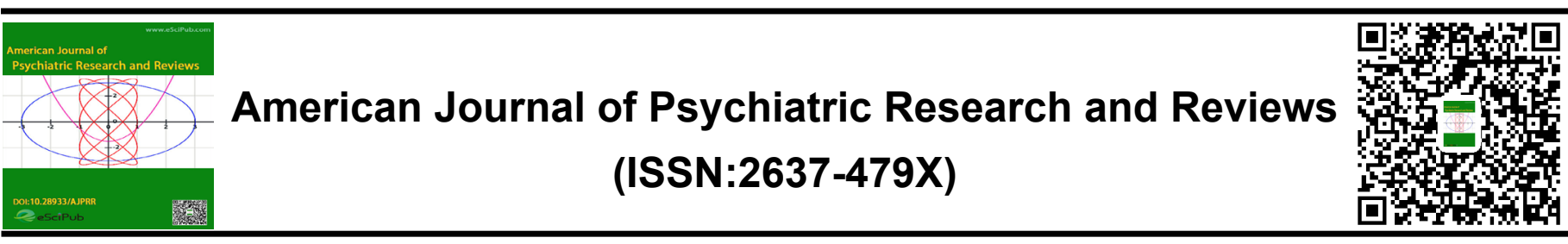

\title{
The Impact of COVID-19 Pandemic in Psychiatric Outpatient Visits in Abu Dhabi - A Retrospective Study
}

\author{
Dr. Aisha Al Dhufairi (MRCPsych), Dr. Eman Al Houti, Dr. Sumaya Al Marzouqi, Dr. Alaa Al \\ Nuaimi, Dr. Alya Al-Midfa and Dr. Amani Hassan (FRCPsych)
}

Sheikh Khalifa Medical City

\begin{abstract}
Introduction: Coronavirus pandemics has affected mental health services around the globe. A marked reduction in psychiatric admission and emergency visits were reported recently. With regards to outpatient services, there was an adoption of tele-psychiatry in mental health facilities in many countries.

Objectives: To examine the impact of COVID-19 pandemics on the psychiatric outpatient visits in the largest Psychiatric Hospital in Abu Dhabi, UAE, during the month of April 2020.

Methodology: A descriptive retrospective study of medical re-

cords of all patients attended the general adult psychiatry clinic in the month of April 2020.
\end{abstract}

*Correspondence to Author:

Dr. Aisha AI Dhufairi (MRCPsych), Sheikh Khalifa Medical City

Results: 1,050 patients were included in the analysis. The sample consists of $55.9 \%$ males. The mean age was 40.7 years. The predominant ethnicity was Arab (88.3\%). The most prevalent diagnosis was depressive disorder $32.7 \%$. Only $1.6 \%$ patients attended the clinic as new appointments. Tele-assessment was conducted in $64 \%$ ( $\mathrm{N}=672$ ) visits. $9.3 \%$ reported to be in relapse during April. Patient with schizophrenia showed the lowest relapse rate $5.7 \%$. Only $8.5 \%$ had taken PCR COVID test, with two patients tested positive.

How to cite this article:

Aisha Al Dhufairi, Eman Al Houti, Sumaya Al Marzouqi, Alaa Al Nuaimi, Alya Al-Midfa, Amani Hassan. The Impact of COVID-19 Pandemic in Psychiatric Outpatient Visits in Abu Dhabi - A Retrospective Study. American Journal of Psychiatric Research and Reviews. 2021; 4:26

Conclusion: The great shift to tele-assessments and the service of medications home delivery were the main reason for maintaining the service in the psychiatric outpatient settings.

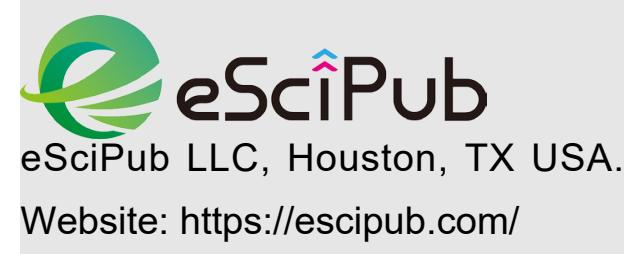




\section{Introduction and background}

The World Health Organization declared the Coronavirus disease [COVID-19] outbreak a Pandemic in March, 2020. [1] Literature have reported direct and indirect impact of the pandemic on both people's physical and mental health. Stress related to the COVID-19 and the social distancing the governments have imposed to decrease community transmission of COVID-19 have worsened mental health in the general population. [2, 3, 4, 5] Furthermore, the COVID-19 negative impact is expected to be higher in people living with mental disorders, due to the vulnerability of those people to stress compared to the general population. They may be more affected by the emotional responses related to COVID-19 Pandemic, and might have increased rate of relapse. ${ }^{[6,7]}$ However, it was reported that the effect of COVID-19 pandemic on patients with mental disorders varies based on different psychiatric diagnoses. Patients with non-psychotic psychiatric illnesses experienced higher level of depression, anxiety, Posttraumatic stress disorder symptoms, insomnia, anger, irritability and suicidal ideation in comparison to healthy controls. ${ }^{[7]}$ While patients with psychotic illnesses reported to be more susceptible to transmission of COVID-19 infection due to illness related cognitive decline, lower awareness of risk, and non-compliant to precautionary measures of infection control. [2, 8]

Evaluating the pandemic effect on mental health services by studying certain indicators, such as rate of hospital admission, outpatient's and emergency department's visits would help understanding the impact of COVID-19 on people with psychiatric illnesses. A marked reduction in psychiatric admission rates during COVID-19 pandemic has been reported in some psychiatric institutes in Italy. ${ }^{[9]}$ Another study, in Portugal, had observed a $52 \%$ relative decrease as well on the total number of psychiatric emergency visits. [10] The immediate impact of COVID-19 should be expected to be greater in outpatient settings, where the majority of mental health care is delivered. The pandemic has provoked unprecedented changes in healthcare delivery in outpatient service. There was a widespread adoption of tele-psychiatry in mental health facilities around the world in response to WHO recommendation of avoiding close contact with others to prevent infection transmission. [11] In some hospitals in the United States; teleassessment increased by $97 \%$ in some psychiatric outpatient departments, which have resulted in decreased no-show rates by $20 \%$ compared to pre-COVID period. [12] In other countries, where tele-assessment cannot be widely implemented due to lack of resources; patients reported to have missed their appointments, stopped their psychiatric medications and eventually relapsed. [8]

Mental health care in the Emirate of Abu Dhabi, the Capital of United Arab Emirates, is provided by two Governmental entities, which are the only available admitting facilities [total of 159 beds] in the Emirate that hosts around 2.9 million populations. [13] Private sector provides only outpatient services. According to $\mathrm{WHO}$ report in 2015 , there is a shortage of mental health professionals number in UAE. There are 1.65 Psychiatrists and 0.52 psychologists per 100,000 populations in Abu Dhabi. Neuropsychiatric disorders contribute to $19.9 \%$ of burden of disease in UAE. Yet, psychiatric disorders are not timely diagnosed in the UAE due to social stigma. [14]

Mental health care is available to everyone in the country, and most of the cost is covered in Public health care sector by the Department of Health. For UAE nationals, who contributes to $19 \%$ of total population of Abu Dhabi, ${ }^{[13]}$ Mental health care is covered in the social insurance scheme in both private and Public sector. [15]

The outpatient department at Behavioral science pavilion [BSP], the largest and the main public psychiatric facility in Abu Dhabi, serves thousands of patients every year; with an average of 2,500 visits in all mental health subspecialties, including psychology, every month. The facility receives referrals from medical and non-medical organizations from 
around the country, hence the average number of newly assessed patients is around 250 patients every month. ${ }^{[16]}$

The United Arab Emirates was the first country in the Middle East to report a confirmed case of COVID-19 in January 2020. Until March 2020, the daily announced cases of positive COVID-19 were still below 50 cases but it reached 12,481 cases by end of April 2020 [17].

Tele-assessment service was never being operated in the facility before the pandemics. However, in response to clear directions from the department of health and hospital management; The workflow at the outpatient clinic have changed dramatically since the last week of March, 2020. Health care providers have received quick technical-operational training on tele-assessment, which was followed by immediate implementation of teleassessment in all psychiatric outpatient services. Face-to-face assessment were only available for new appointment patients and patients who in-person evaluation was deemed clinically necessary. Moreover, medications were delivered home by mental health professionals [nurses and occupational therapists] who can administer long acting injections at home as well. Despite all the above mentioned measures to maintain the service in the outpatient clinic; there was a declined rate of $20 \%$ in the total number of patients registered [both for face-to-face and tele-assessment visits] in psychiatric outpatient clinic including all subspecialty clinics. ${ }^{[16]}$

In this context, we have examined the impact of COVID-19 pandemic in psychiatric outpatient department in behavioral science pavilion [BSP] during April 2020, the beginning of the pandemics.

\section{Methodology:}

A descriptive retrospective study of medical records of all patients attended the general adult psychiatry clinic at Behavioral Science Pavilion [BSP], in the month of April 2020. Ethical approval from the Institutional Review Board
[IRB] in the Department of Health [DOH] of Abu Dhabi was obtained. Exclusion criteria were Patients aged 65 year and below 18 year-old, Forensic cases, substance misuse, neurodevelopmental, neuro-cognitive disorders and patient who did not attend their appointments. The data, which was obtained from patients' electronic records included patient's age, gender, level of education, occupation, marital status, psychiatric and medical diagnoses, COVID-19 status, type of visit and outcome. The type of visit was classified into face-to-face or tele-assessment and each further divided into new or follow up. The outcome of each visit was reported either relapsed, improved or stable, based on physicians' documentation in patients electronic files. Data cleaning for $10 \%$ of the sample was done repeatedly until no more mistakes were found. The data were analyzed using SPSS version 26 to establish basic statistics of the variables. Data were presented as frequencies and percentages for categorical data.

\section{Results}

A total of 1,439 Medical electronic records for all patients attended the general adult clinic in April 2020 were examined. Out of which, 1,050 patients' visits were included in the analysis.

\section{Demographic characteristics}

The sample consists of $55.9 \%$ [ $N=587]$ males and $44.1 \%$ [ $\mathrm{N}=463$ ] females aged $18-<65$ years, with mean age of [40.7 years and11.725 SD]. More than half of the sample were married [57.5\%]. Marriage was equally more common in both females and males groups in our sample. The sample was predominantly Arab in origin [88.3\%], followed by Asian [9\%], African [1.5\%] and Caucasians [1.2\%]. There was $47 \%$ missing data in occupational status and $66 \%$ missing in Educational level, in patients' medical records, so data were not included.

\section{Visits types}

Chart [1] showed the number and type of clinic visits during April, 2020. Only $1.6 \%$ [N=17] patients attended the clinic as new 
assessments, while $98 \%$ [N=1,033] were known to the service and had either tele-assessment visits [telephone call] 65\%, face-to-face visits $15.8 \%$ or walk-in visits $19 \%$.

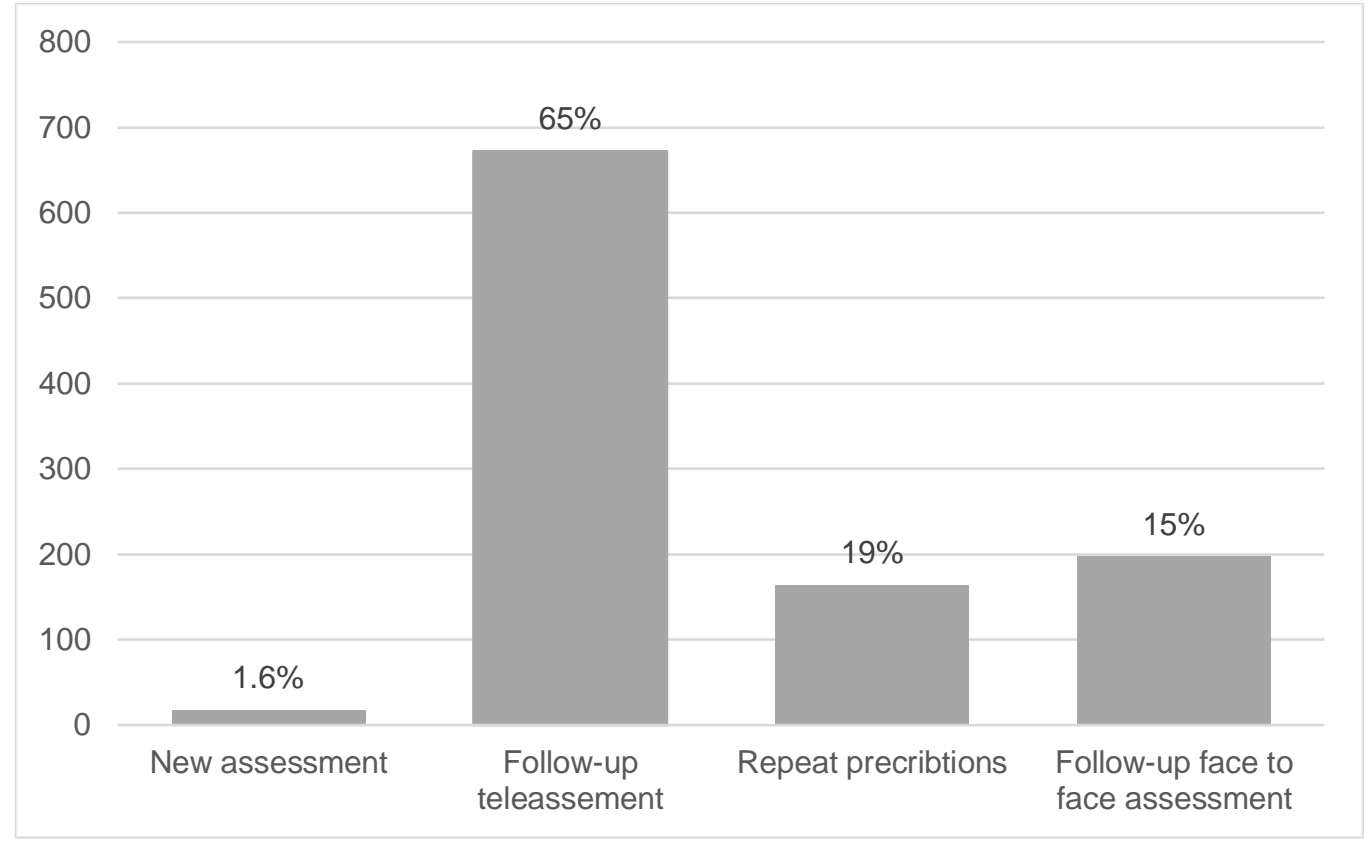

\section{Psychiatric and Medical diagnoses}

for new assessment cases; The most common

The most prevalent diagnoses in our sample was depressive disorders [32.7\%] and the least common was gender dysphoria disorder [0.1\%]. presentations were anxiety [41\%] and depressive [35\%] symptoms followed by psychosis [23.5\%].

With regard to psychiatric presentations specific

Table 1 shows the prevalence of DSM-5 psychiatric diagnoses.

\begin{tabular}{|l|c|}
\hline Psychiatric Diagnoses & Number and Frequency \\
\hline Depressive Disorders & $\mathrm{N}=343[32.7 \%]$ \\
\hline Schizophrenia Spectrum and Other Psychotic Disorders & $\mathrm{N}=277[26.4 \%]$ \\
\hline Bipolar and Related Disorders & $\mathrm{N}=230[21.6 \%]$ \\
\hline Anxiety Disorders & $\mathrm{N}=141[13.4 \%]$ \\
\hline Obsessive-Compulsive and Related Disorders & $\mathrm{N}=48[4.6 \%]$ \\
\hline Personality Disorders & $\mathrm{N}=10[1 \%]$ \\
\hline Gender Dysphoria & $\mathrm{N}=1[0.1 \%]$ \\
\hline
\end{tabular}

With regard to chronic medical conditions chronic medical conditions and taking non[Diabetes mellitus, hypertension and thyroid psychotropic medications. 3.4\% [N=36.75] of the diseases]; $35 \%$ [ $N=368]$ were having comorbid patients have a diagnosis of epilepsy. $45 \%$ of AJPRR:https://escipub.com/american-journal-of-psychiatric-research-and-reviews/ 4 
patients with depressive disorders have comorbid medical conditions, while around 30\% of patients with schizophrenia spectrum or bipolar disorders had comorbid medical diagnoses.

\section{Visits outcome}

Majority of the sample; $89.9 \%$ [N=944], reported to be stable during the assessment. $9.3 \%$ $[\mathrm{N}=98]$ required treatment or change of medications $7.6 \%[\mathrm{~N}=80]$, or inpatient admission $1.7 \%[\mathrm{~N}=18]$. Only $0.76 \% \quad[\mathrm{~N}=8]$ patients reported improvement of symptoms. Relapse rate was higher in face to face assessment group compared to tele-assessment group; $21.8 \%$ and $4 \%$ respectively.

Out of those who relapsed and needed intervention, majority had depressive disorders [33\%] followed by bipolar disorder [25\%]. The relapse rate among psychiatric diagnoses groups was the highest in patients with personality disorders [20\%], followed by obsessive compulsive disorder [16.6\%], anxiety disorders [12.7\%], in bipolar disorders [10.8\%], in depressive disorder [8.5\%] and the lowest relapse rete was in schizophrenia spectrum group [5.7\%].

Table 2 shows the outcome of visits in relation to type of visits.

\begin{tabular}{|l|c|c|c|c|c|}
\hline $\begin{array}{l}\text { Table [2] Type of assessment } \\
\text { and visits outcome }\end{array}$ & Stable & $\begin{array}{c}\text { Required treatment or } \\
\text { change of medication }\end{array}$ & Admitted & improved & Total \\
\hline New Case & 0 & 16 & 1 & 0 & $17[1.6 \%]$ \\
\hline Follow up: Face to face & 154 & 37 & 5 & 1 & $197[18.8 \%]$ \\
\hline Follow up: tele-assessment & 639 & 19 & 8 & 6 & $672[64 \%]$ \\
\hline Walk-in No Appointment & 151 & 8 & 4 & 1 & $164[15.6 \%]$ \\
\hline Total & $944[89.9 \%]$ & $80[7.6 \%]$ & $18[1.7 \%]$ & $8[0.76 \%]$ & 1050 \\
\hline
\end{tabular}

\section{COVID-19 test and results}

By April, only 89 patients [8.5\%] had taken PCR COVID test, and only two patients tested positive in our sample. $21.4 \%$ of patients who reported to be in relapse in their symptoms, and required intervention, had taken PRC test voluntarily, while only $7.2 \%$ of those who reported stable condition had taken the test. With respect to psychiatric diagnoses and PRC test; we found that only $3.6 \%$ of patients with schizophrenia, 9.1 $\%$ of patients with bipolar disorder, $10.7 \%$ of patient with depressive disorder, $14.9 \%$ of patients with anxiety disorders, and $4.6 \%$ of patients with Obsessive compulsive disorders took PRC test voluntarily.

\section{Discussion}

- Sample characteristics
Males outnumbered females, which correlates with UAE gender dominance of male gender [72\%]. Although Indian subcontinent expats are the majority in the country [59.48\%]; $88 \%$ of our sample were of Arab origin. This raised the concern about the mental health services for this population. Moreover, most of this group of population are of labor workers who usually get deported back to their countries if found to have severe mental disorders.

There was no change in the frequencies of different psychiatric disorders in the clinic before and during COVID-19. [16] This could be explained by tele-assessment service that was implemented and outreached to majority of appointment patients since the beginning of the pandemic. Our findings were similar to 
international studies in regards to psychiatric disorders frequencies in psychiatric outpatient clinics. Studies [18] [19] have shown that the majority of follow-up patients in psychiatric facilities have diagnoses of sever and enduring mental disorders such as schizophrenia, schizoaffective and bipolar affective disorders. Comparing to study in the same region; Saudi Arabia, we showed the same results of the most common psychiatric diagnoses among outpatients were major depressive disorder and schizophrenia. [19]

\section{- Clinic visits types and numbers}

An overall declined rate of $20 \%$ was noticed in the number of patients registered in psychiatric outpatient clinic including general and subspecialty clinics. In fact, the great percentage of decline in number of visits were noticed in the geriatric, child and adolescent psychiatry clinics, which were not studied in this context. But with specification to general adult psychiatry outpatient services, there is only $5-10 \%$ drop rate in clinic visits number in April 2020, in comparison with previous months of the same year; mainly in new assessment visits. [16] Nevertheless, that is only true if we consider digital communication with patients [telephone calls] as an actual clinic visit. As teleassessment was not offered to new-appointment patients in our facility; there was significant drop, of $90-94 \%$, in visits number compared to previous months of the same year [17 cases compared to 250 cases per month previously]. [16] The drop in new assessment visits can be explained by increasing the threshold of seeking psychiatric care in the community; patients and family members may have become more tolerant during the epidemic, avoiding hospitals, and the risk of contamination, although the COVID-19 pandemic should have caused a parallel epidemic of fear and anxiety in the community. Additionally, the curfew and the massive quarantine between the major cities in the country might have affected the access to the facility. ${ }^{[8]}$
With regard to visits assessment types; There was $80 \%$ drop rate in face-to-face visits and massive implementation of tele-assessment services $+600 \%$ compared to Pre-COVID-19 months. Furthermore, although there is no drop of walk-in visits number compared to PreCOVID-19 months, hospital administration allowed patients who had no appointments [probably missed their earlier scheduled appointments and prescriptions] to send a family member on their behalf to register in clinic and collect the medications from the pharmacy. This change in clinic policy was aimed at reducing the risk of infection to vulnerable population of mental disorders who have proposed reduced body immunity. ${ }^{[21,22]}$ When tele-assessment and medications home delivery were initiated by the health care facility; patients don't have to deal with their fear of exposure to the infection. In fact, in comparison with another study on psychiatric outpatient visits during COVID-19 which showed that only $12.8 \%$ could contact mental health professionals either directly or through tele-consultation, and 22\% stopped their psychiatric medications due to enforcement of lockdown or lack of transportation due to COVID-19. [12]

\section{- Relapse rate during COVID-19}

Relapse rate was $9 \%$ in the sample. In the relapsed group, majority of patients had diagnoses of depressive disorders, which was the most prevalent diagnosis in the sample. However, by studying relapse rate within each diagnostic group; it showed that patients with personality disorders were having the highest rate of relapse followed by obsessive compulsive disorder and anxiety disorders. While patients with schizophrenia showed the lowest relapse rate. Similarly, a study that had examined the psychiatric emergency room visits during COVID-19 concluded that personality disorders were among the diagnoses that showed increased in emergency room visits. ${ }^{[9]}$ owing to their mood instability and underlying hyper-responsiveness to stress, often associated with acting-out behaviors; drug 
intoxications and substance consumption. Another studies have also confirmed that patients with anxiety and depressive disorders may be more affected by the emotional responses related to COVID-19 pandemic, and might have increased rate of relapse. ${ }^{[6,7]}$ In contrast to patients with psychotic disorders who showed to have less awareness and concerns about the pandemics. ${ }^{2,8]}$

Relapse rate was higher in the group who showed for their appointment face-to-face and did not prefer tele-assessment visits; $21.8 \%$ compared to only $4 \%$ who had tele-assessment visits. That indicates that patients who are known to the facility came to clinic or brought by family for in-person evaluation if they were having active symptoms of mental disorders. This attitudes of patients raised the question of the effectiveness and the acceptance of teleassessment in replacing face-to-face assessment by psychiatric patients. Many studies have examined the effectiveness of teleassessment in psychiatry practice. Establishing rapport has been a challenge, as many psychiatrists reported that technology does not replace in-person interaction. Treatments relying heavily upon non-verbal communications [e.g., insight-oriented/relational individual and group psychotherapy] require unique adaptation and adjustment. Certain Patients with paranoia or other psychotic symptoms, with auditory/visual impairment, or with cognitive impairment may also be uniquely challenged. ${ }^{[11]}$

\section{- COVID-19 PCR test}

The percentage of patients who took COVID-19 PRC test was only $8.5 \%$. In April, the pandemic had not peaked yet in the country, so most people might not be keen to take COVID-19 tests. Out of those who took the test, the minority were having a diagnosis of schizophrenia spectrum [13\% only]. Moreover, relapse rate was the lowest in patients with schizophrenia spectrum [5.7\%]. This could be explained by the level of awareness and the insight of people with severe mental disorder about the seriousness of the pandemics. A study in India showed that three-forth of patients with severe mental disorders were not worried about getting COVID-19 and lacks adequate knowledge to identify symptoms. Two-third of patients lacked adequate knowledge of precautionary measures against COVID-19. ${ }^{[8]}$ Where in developed countries with greater resources and more established health care system; In UK individuals with psychiatric disorders have been tested for COVID-19 more frequently than individuals without a psychiatric history. Possibly, people with psychiatric disorders are being tested more frequently because of co morbid conditions, higher levels of anxiety about contracting COVID-19 or perhaps a combination of both. [20]

In conclusion, the great shift to teleassessments and the service of medications home delivery were the main reasons for maintaining the service in the outpatient settings during a pandemic. When tele-assessment service was not offered to new-assessment appointments, there was a great drop in newly assessed cases in the facility. Hence, hospital regulations should permit tele-assessment service for both established and new cases in the future. Patients with schizophrenia spectrum disorders showed to be the least aware about the pandemics. We recommend special attention and care for this group during similar pandemics. Further studies in this area are required to examine the impact of a health pandemics in psychiatric outpatient activities.

\section{Study limitations}

The study only examined the impact of the pandemic during the month of April, when the pandemics was not peaked yet in the country, so our finding might not be accurate enough to establish the true effect of pandemics on psychiatric patients' attitudes in outpatient clinic. Moreover, the services of tele-assessment and medications home delivery have masked the true effect of pandemics on the outpatient services. Researchers have chosen the predominant psychiatric diagnoses and not included the other comorbid psychiatric 
diagnoses, which might explain the low prevalence of personality disorders.

\section{Study Strength}

The sample size was good, which includes all adults with general psychiatric disorders who attended the service. The study is the first in the Middle East to report the effect of COVID 19 on psychiatric outpatient services. The sample also has a wide range of ethnic groups, which reflects the demographic of Abu Dhabi.

\section{Declaration of Interest}

None of the authors has a grant support, other external funding or any other conflict of interest.

\section{References}

[1]. Jiiang S, Shi Z, Shu Y, Song J, Gao G, Tan W, Guo D. A distinct name is needed for the new coronavirus. The Lancet [Internet]. 2020, March [cited 2020, Feb 19] .Volume 395, P949. Available from: https://doi.org/10.1016/S01406736[20]30419-0

[2]. Kozloff N, Mulsant B, Stergiopoulos V, Voineskos A1. The COVID-19 Global Pandemic: Implications for People with Schizophrenia and Related Disorders. Schizophrenia Bulletin [internet]. 2020 July [cited 2020 April 29]. Volume 46, Issue 4, P 752-757.

Available from: https://doi.org/10.1093/schbul/sbaa051.

[3]. AlKhamees A, Alrashed S, Alzunaydi A, Almonhimeed A, Aljohani M. The psychological impact of COVID-19 pandemic on the general population of Dausi Arabia. Comprehensive psychiatry [internet]. 2020. 102[2020] 152192. Available

from: https://doi.org/10.1016/j.comppsych.2020.15219 2

[4]. Thomas J, et al. Psychosocial Correlates of Depression and Anxiety in the United Arab Emirates During the COVID-19 Pandemic. Frontiers in Psychiatry [internet]. 2020; 11:564172. Available from: https://www.ncbi.nlm.nih.gov/pmc/articles/PMC7 683430/

[5]. Tee M, et al. Psychological impact of COVID-19 pandemic in the Philippines. J Affect Disord [Internet]. 2020 December. P 379-391. Available from:

https://www.ncbi.nlm.nih.gov/pmc/articles/PMC7 444468/

[6]. Ya H, Chen J, Xu Y. Patients with mental health disorders in the COVID-19 epidemic. The Lancet
Psychiatry [Internet]. 2020, April [cited 2020 April 1]. Volume 7 [ISSUE 4, e21]. Available from: https://doi.org/10.1016/S2215-0366[20]30090-0 E2

[7]. Hao F, et al. Do psychiatric patients experience more psychiatric symptoms during COVID-19 pandemic and lockdown? A case-control study with service and research implications for immunopsychiatry. Brain, behavior and immunity [Internet]. 2020. Available from: https://www.promis.scot/wpcontent/uploads/2020/05/Hao-et-al-2020-Dopsychiatric-patients-exerience-more-psychiatricsymptoms-during-CV19-pandemic-andlockdown-2.pdf

[8]. Muruganandama P, Neelamegama S, Menonb V, Alexandera J, Chaturvedi S. COVID-19 and Severe Mental Illness: Impact on patients and its relation with their awareness about COVID-19. Psychiatry Res J [internet]. 2020 Sepember [cited 2020 Jun 29]. P 291:113265. Available from: https://www.ncbi.nlm.nih.gov/pmc/articles/PMC7 322460/

[9]. Clerici M, Durbano F, Spinogatti F, Vita A, de Girolamo G. Psychiatric hospitalization rates in Italy before and during COVID-19: did they change? An analysis of register data. Ir $\mathrm{J}$ of Psychol Med [internet]. 2020 May. 10.1017/ipm.2020.29. available from: https://www.ncbi.nlm.nih.gov/pmc/articles/PMC7 264453/

[10]. Gonçalves-Pinho M, Mota P, Ribeiro J, Macedo S, Freitas A. The Impact of COVID-19 Pandemic on Psychiatric Emergency Department Visits - A Descriptive Study. Psychiatric Quarterly. 2020 August [published 2020 Aug 25]. Available from: https://doi.org/10.1007/s11126-020-09837-z.

[11]. World health Organization. Coronavirus disease [COVID-19] advice for the public [internet]. 2020 [updated 2020 Dec 8] Available from: at:https://www.who.int/emergencies/diseases/nov el-coronavirus-2019/advice-for-public.

[12]. A. Chen J et al. COVID-19 and telepsychiatry: Early outpatient experiences and implications for the future. General hospital psychiatry journal [internet]. 2020 [cited September-October 2020]. Volume 66. P 89-95. Available from: https://linkinghub.elsevier.com/retrieve/pii/S0163 834320301031

[13]. Wikipedia. Abu Dhabi. 2020 [updated 2020 Dec 1]. Available at: https://en.wikipedia.org/wiki/Abu_Dhabi.

[14]. World Health Organization. United Arab Emirates Mental Health. 2010. Available at: 
https://www.who.int/mental_health/evidence/atla s/profiles/are_mh_profile.pdf?ua $=1$.

[15]. World Health Organization. Regional Office for the Eastern Mediterranean. Country cooperation strategy for WHO and United Arab Emirates: 2012 - 2017. World Health Organization, Regional Office for the Eastern Mediterranean [internet]. P7. Available from: https://apps.who.int/iris/handle/10665/113226

[16]. BSP portfolio. Sheikh Khalifa Medical City [internet]. 2020 [updated Nov 30]. Available at: http://portal.seha.ae/SKMC/sites/bsp/Quality\%20 Department/Occurance\%20Portfolio\%202009/Fo rms/Allltems.aspx

[17]. WorldOMeter. United Arab Emirates. 2020. Available at: https://www.worldometers.info/coronavirus/countr y/united-arab-emirates/

[18]. Killaspy H, Banerjee S, Lloyd M, King M . A prospective controlled study of psychiatric outpatient non-attendance. Characteristics and outcome. Br J Psychiatry [internet]. 2000 Febrauary. Volume 176 , Issue 2 , pp. 160 - 165 DOI. Available from: https://www.cambridge.org/core/product/identifier /S000712500026412X/type/journal_article

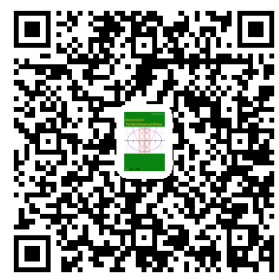

[19]. Al Osaimi F. et al. Patterns of psychiatric diagnoses in inpatient and outpatient psychiatric settings in Saudi Arabia. Archives of Clinical Psychiatry. Arch Clin Psychiatry [Internet]. 2017;44[3]:P 77-83.

[20]. Der Meer D , Pinzón-Espinosa J, D. Lin B, K. Tijdink J, Vinkers C, Guloksuz S, J. Luykx J. Associations between psychiatric disorders, COVID-19 testing probability and COVID-19 testing results: findings from a population-based study. BJPsych Open. 2020 September [Cited 2020 July 22]. Volume 6, Issue 5, e87. Available from:

https://www.cambridge.org/core/journals/bjpsychopen/article/associations-between-psychiatricdisorders-covid19-testing-probability-andcovid19-testing-results-findings-from-apopulationbased-study.

[21]. Seminog OO, Goldacre MJ. Risk of pneumonia and pneumococcal disease in people with severe mental illness: English record linkage studies. Respiratory epidemiology [Internet]. 2012 December. Available from: http://thorax.bmj.com/

[22]. Oliveira J, Oliveira-Maia AJ, Tamouza R, Brown AS, Leboyer M. Infectious and immunogenetic factors in bipolar disorder. Acta Psychiatrica Scandinavica [Internet]. 2017. Vol. 136, Blackwell Publishing Ltd; 2017. p. 409-23. 